University of Nebraska - Lincoln

DigitalCommons@University of Nebraska - Lincoln

July 1998

\title{
Chemical Synthesis of Nanostructured Cobalt at Elevated Temperatures
}

Diandra Leslie-Pelecky

University of Nebraska -- Lincoln, diandra2@unl.edu

M. Bonder

University of Nebraska - Lincoln, NE 68588-0111

T. Martin

University of Nebraska - Lincoln, NE 68588-0111

E.M. Kirkpatrick

University of Nebraska - Lincoln, NE 68588-0111

X.Q. Zhang

University of Nebraska - Lincoln, NE 68588-0111

See next page for additional authors

Follow this and additional works at: https://digitalcommons.unl.edu/physicslesliepelecky

Part of the Physics Commons

Leslie-Pelecky, Diandra; Bonder, M.; Martin, T. ; Kirkpatrick, E.M.; Zhang, X.Q.; Kim, S.-H.; and Reike, Reuben D., "Chemical Synthesis of Nanostructured Cobalt at Elevated Temperatures" (1998). Diandra LesliePelecky Publications. 13.

https://digitalcommons.unl.edu/physicslesliepelecky/13

This Article is brought to you for free and open access by the Research Papers in Physics and Astronomy at DigitalCommons@University of Nebraska - Lincoln. It has been accepted for inclusion in Diandra Leslie-Pelecky Publications by an authorized administrator of DigitalCommons@University of Nebraska - Lincoln. 


\section{Authors}

Diandra Leslie-Pelecky, M. Bonder, T. Martin, E.M. Kirkpatrick, X.Q. Zhang, S.-H. Kim, and Reuben D. Reike 


\title{
Chemical Synthesis of Nanostructured Cobalt at Elevated Temperatures
}

\author{
Diandra L. Leslie-Pelecky, M. Bonder, T. Martin, E.M. Kirkpatrick, X.Q. Zhang, S.-H. Kim and Reuben D. Rieke \\ University of Nebraska, Lincoln, NE 68588-0111
}

\begin{abstract}
Chemical synthesis is a versatile technique for fabricating novel nanostructured materials. In the Rieke process, a metal salt is reduced by an alkali in a hydrocarbon solvent to form small, highly reactive particles. Synthesis at an elevated temperature $\left(200^{\circ} \mathrm{C}\right)$ increases the as-synthesized particle size and produces higher coercivities and remanence ratios than observed in similar syntheses at room temperature. The ratio of synthesis temperature to solvent boiling point appears to be an important parameter in both coercivity and oxidation resistance.
\end{abstract}

Index Terms - chemical synthesis, cobalt, magnetic properties of nanostructured materials

\section{INTRODUCTION}

Nanostructured materials often have unique electrical, chemical, structural and magnetic properties due to a combination of the reduced grain size and large surface or interface area relative to bulk forms of the same material. The number of fabrication techniques and potential applications for nanostructured materials has grown significantly[1,2] since their initial discovery. Chemical synthesis requires little specialized equipment and can generate large quantities of materials. The as-synthesized materials generally have very small $(<5 \mathrm{~nm})$ crystallites, although larger grain sizes can be produced by annealing. Although organic fragments may be incorporated during synthesis, reactions between these fragments and the metal during annealing often produce metastable materials with novel magnetic behavior.

\section{SAMPLE FABRiCATION AND MEASUREMENT}

In 1972, Rieke and co-workers reported a general approach for preparing highly reactive metal powders using alkali metals to reduce metal salts in ethereal or hydrocarbon solvents. $[3,4]$ Cobalt nanoparticles are produced by:

$$
\mathrm{CoI}_{2}+2 \mathrm{Li} \longrightarrow \mathrm{Co}^{*}+2 \mathrm{LiI}
$$

The reaction occurs in a solvent in which the salt has at least partial solubility. An electron carrier such as naphthalene facilitates electron charge transfer. The LiI generated in the reduction can be removed by washing the product with an appropriate solvent after synthesis, or retained to reduce interparticle interactions. The standard synthesis is performed at room temperature. [5]

Synthesis at elevated temperatures may be desirable for

Manuscript received October 16, 1997

Diandra L. Leslie-Pelecky 402-472-9178, fax: $402-472-2879$ diandra@unlinfo.unl.edu

This work was supported in part by the Nebraska Research Initiative through the Center for Materials Research and Analysis at the University of Nebraska and the National Science Foundation under grants OSR-925525 and DMR-9424018. three reasons. First, higher temperatures eliminate the need for an electron carrier, which can change reaction rates. Second, the coercivity and remanence ratios of nanostructured materials are strongly dependent on crystallite size. Varying the synthesis temperature allows direct control of the assynthesized crystallite size, eliminating the need for postsynthesis annealing. Finally, high-temperature synthesis decreases reactivity and thus reduces oxidation.

Table I summarizes the synthetic protocols. The yield ratio is the ratio of the weight of the final material to the weight expected from (1) assuming that all of the lithium iodide is removed.

Run 80 used the standard room-temperature synthesis with THF as the solvent and a molar ratio of $5 \%$ naphthalene to lithium. Run 80 was not washed after synthesis, which is reflected in the large yield ratio.

Runs 81,82 and 83 were synthesized at temperatures of $200^{\circ} \mathrm{C}$ using dodecane $\left(\mathrm{CH}_{3}\left(\mathrm{CH}_{2}\right)_{10} \mathrm{CH}_{3}, \mathrm{bp}=216^{\circ} \mathrm{C}\right)$, pentadecane $\left(\mathrm{CH}_{3}\left(\mathrm{CH}_{2}\right)_{13} \mathrm{CH}_{3}, \mathrm{bp}=271{ }^{\circ} \mathrm{C}\right)$ and undecane $\left(\mathrm{CH}_{3}\left(\mathrm{CH}_{2}\right)_{9} \mathrm{CH}_{3}, \mathrm{bp}=196^{\circ} \mathrm{C}\right)$ as solvents. A THF wash was used to remove the Lil, resulting in yield ratios close to one. The lower yield ratio of Run 83 is due to the strong magnetism of the sample, which hinders removal of the sample from the magnetic stir bar used in the synthesis.

TABLE I

SUMMARY OF SAMPIES SYNTHESIZED

\begin{tabular}{llrc}
\hline$\#$ & Solvent & Yield Ratio & Washed in \\
\hline 80 & THF & 6.63 & no wash \\
81 & n-dodecane & 1.00 & THF \\
82 & n-pentadedecane & 1.03 & THF \\
83 & n-undecane & 0.86 & THF \\
\hline
\end{tabular}

$X$-ray diffraction measurements were performed in a Rigaku D-Max B diffractometer. The diffracting crystallite size (DCS) is obtained from the Scherrer equation, in which the breadth, $\mathrm{B}$, of a diffraction peak located at $2 \theta$ is related to the diffracting crystallite size by

$$
\mathrm{DCS}=\frac{0.9 \lambda}{\mathrm{B} \cos \theta}
$$

where $\lambda$ is the $\mathrm{x}$-ray wavelength. XRD measurements are made by placing the particles in a small amount of silicone grease on the surface of a low-background quartz holder.

Scanning Electron Microscopy (SEM) and Transmission Electron Microscopy (TEM) show that all of the protocols produce micron-sized agglomerates with DCSs from 5 to $50 \mathrm{~nm}$ depending on annealing treatment. EnergyDispersive X-Ray Spectroscopy (EDX) confirms that most samples retain a small amount of iodine, even after washing.

Magnetic properties are measured using an alternating gradient force magnetometer. Powder is placed in a paraffin- 


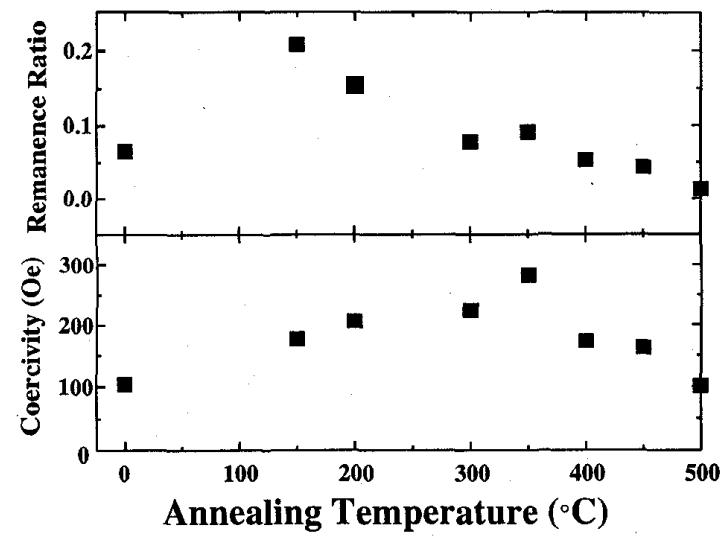

Fig. 1. Dependence of the coercivity $\left(H_{c}\right)$ and remanence ratio $\left(M_{r} / M_{s}\right)$ on the annealing temperature. All samples were annealed for one hour.

filled polyethylene bag and sealed under an inert atmosphere. The polyethylene bag prevents oxidation during the measurements and the paraffin immobilizes the particles to prevent whole-particle rotation.

\section{RESULTS AND DISCUSSION}

\section{A. Room-Temperature Synthesis}

The x-ray diffraction pattern of the as-synthesized nanoparticles shows only very broad diffraction peaks corresponding to $\mathrm{CoO}$, suggesting oxidation on exposure to air. Oxidation likely occurs during the XRD measurement, as diffraction patterns from annealed samples show less evidence of CoO. Sample reactivity is known to decrease after annealing due in part to the decreased surface area. XRD patterns from samples annealed at temperatures above $350^{\circ} \mathrm{C}$ show peaks due to both hexagonal and cubic forms of cobalt. Samples annealed at lower temperatures $\left(<350^{\circ} \mathrm{C}\right)$ show evidence of $\mathrm{CoO}$, which we again attribute to oxidation during the XRD measurement.

The coercivity and remanence ratio vary with annealing temperature, as shown by Fig. 1. All samples were annealed for one hour. Coercivities range from $100 \mathrm{Oe}$ to $\approx 300 \mathrm{Oe}$ and remanence ratios range from 0.08 to 0.18 .

\section{B. High-Temperature Synthesis}

The differences between the high-temperature and lowtemperature syntheses are illustrated in Fig. 2. In contrast to the low-temperature-synthesized material, all of the hightemperature syntheses exhibit well-defined peaks at the positions corresponding to hexagonal cobalt.

The cobalt peaks at $41.7^{\circ}(100)$ and $47.6^{\circ}(101)$ are analyzed using (2) to obtain DCS values. The peak at $44.8^{\circ}$ is not used, as there is a possible overlap with a peak due to fcc cobalt. The DCSs of as-synthesized powders range from $7( \pm 3) \mathrm{nm}$ for the dodecane sample to $9.7( \pm 1 \mathrm{~nm})$ for the undecane materials as calculated from the peak at $47.6^{\circ}$. The peak positions of the high-temperature-synthesized samples are shifted $\left(0.1^{\circ}-0.4^{\circ}\right)$ to lower values than expected, with the greatest shift in the undecane-synthesized sample.
Differential broadening of the XRD peaks is observed in all samples. The peak at $41.7^{\circ}$ provides a DCS greater than that of the DCS calculated from the peak at $47.6^{\circ}$. The difference is smallest in the unannealed samples (within a factor of two) and increases as samples are annealed. Differentiall broadening could be due to an overlap with a yetunidentified peak; however, strain introduced as the materials cool from the synthesis temperature could also produce differential broadening. The pattern of the broadening is consistent with the presence of fcc stacking faults. Highresolution TEM will be performed to determine whether strain is responsible for the differential broadening.

Broad $\mathrm{CoO}$ peaks are present in the XRD pattern of the dodecane-synthesized sample, but not in the other two diffraction patterns. Comparison with the XRD pattern from Run 80 shows that the high-temperature synthesis produces less reactive particles with improved stability against oxidation. Table II compares the coercivity and remanence ratios of the as-synthesized particles.

TABLE II

\begin{tabular}{rlrc}
\hline$\#$ & Solvent Formula & Coercivity (Oe) & Remanence Ratio \\
\hline 80 & $\mathrm{C}_{4} \mathrm{H}_{8} \mathrm{O}$ & 96 & 0.03 \\
82 & $\mathrm{CH}_{3}\left(\mathrm{CH}_{2}\right)_{13} \mathrm{CH}_{3}$ & 162 & 0.12 \\
81 & $\mathrm{CH}_{3}\left(\mathrm{CH}_{2}\right)_{10} \mathrm{CH}_{3}$ & 195 & 0.13 \\
83 & $\mathrm{CH}_{3}\left(\mathrm{CH}_{2}\right)_{9} \mathrm{CH}_{3}$ & 534 & 0.33 \\
\hline
\end{tabular}

Although the high-temperature runs have very similar DCSs, the magnetic properties are very different. The coercivity and remanence ratio decrease as the molecular weight of the solvent increases. The sample synthesized in undecane (Run 83) has a significantly larger coercivity and remanence ratio then either Run 81 or Run 82 .

Our studies of chemically synthesized nickel show that the coercivity increases rapidly with increasing DCS up to $20 \mathrm{~nm}$ and then plateaus.[5] Slight differences in DCS could be responsible for the different magnetic properties in different cobalt syntheses; however, samples from different runs with the same DCS still exhibit significantly different magnetic behavior. We therefore conclude that the magnetic properties of the materials are not determined strictly by the DCS.

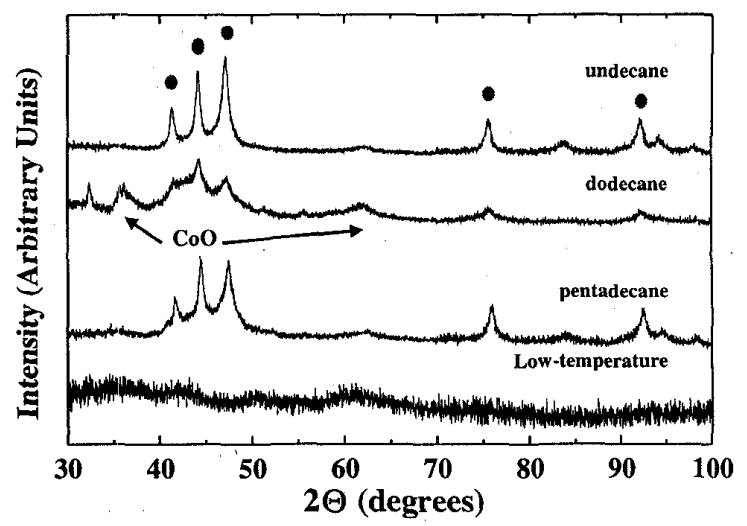

Fig. 2. XRD patterns for as-synthesized samples from (top to bottom) Runs $83,81,82$ and 80 . 


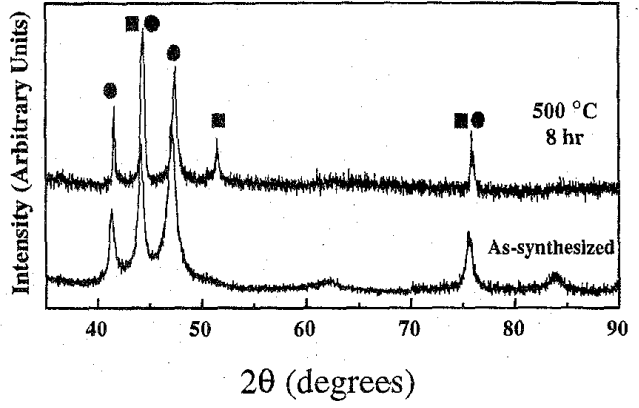

Fig. 3. Comparison of XRD patterns from Run 83. The as-synthesized sample is the bottom pattern and a sample annealed for 8 hours at $500^{\circ} \mathrm{C}$ is the top pattern. Circles denote peaks corresponding to hexagonal cobalt. Squares represent fcc cobalt peaks.

\section{Annealing of High-Temperature-Synthesized Samples}

Samples were annealed at temperatures from $150^{\circ} \mathrm{C}$ to $700{ }^{\circ} \mathrm{C}$ to vary the DCS. Peaks corresponding to the hightemperature fcc cobalt phase were observed after annealing at temperatures above $350^{\circ} \mathrm{C}$ in all of the samples; however, Run 83 exhibits significant amounts of the cubic cobalt phase only when annealed at $500^{\circ} \mathrm{C}$ or above. Fig. 3 compares the unannealed XRD pattern from Run 83 with the pattern of a sample annealed at $500^{\circ} \mathrm{C}$ for 8 hours. The circles denote hcp cobalt peaks and the squares denote fcc peaks.

Annealing increases the differential broadening of the XRD peaks. In Run 83 , the DCS from the peak at $47.6^{\circ}$ changes from $9.7 \mathrm{~nm}$ to a maximum value of $19.4 \mathrm{~nm}$ when annealed at $700^{\circ} \mathrm{C}$. The DCS from the peak at $41.7^{\circ}$, however, changes from $16 \mathrm{~nm}$ to $50 \mathrm{~nm}$ over the same range of annealing temperatures. The other runs behave similarly.

Fig. 4 shows the dependence of the remanence ratio and coercivity on the DCSs obtained from the two XRD peaks. The open symbols represent samples in which fcc cobalt is observed. No significant amounts of cubic cobalt are observed in the samples represented by solid symbols. Fig, 4 shows the correlation between the DCS and the magnetic properties, with the coercivity and remanence increasing with decreasing DCS for the peak at $41.7^{\circ}$. The coercivity and

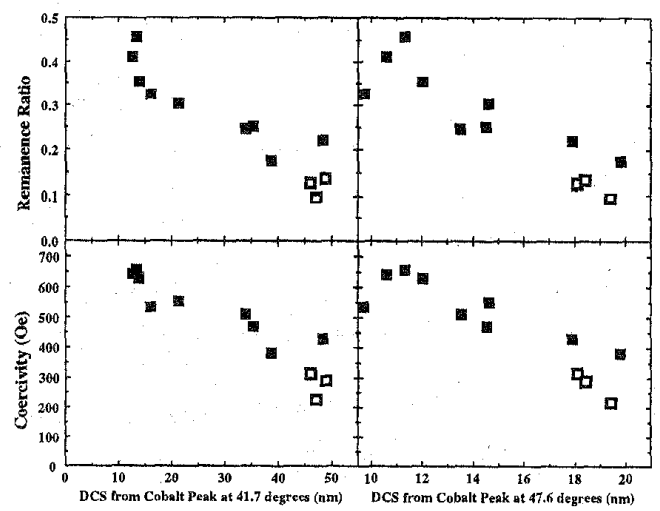

Fig 4. Dependence of coercivity and remanence ratio on the DCSs obtained from the cobalt peaks at $41.7^{\circ}$ and $47.6^{\circ}$ remanence increase to reach a maximum near $11 \mathrm{~nm}$ for the DCS obtained from the peak at $47.6^{\circ}$ and decrease with increasing DCS thereafter. Preliminary electron diffraction measurements show that individual particles exhibit a preferential orientation, suggesting that conditions from growth may be different for different planes. Further analysis of this dependence must wait until the mechanism for the differential broadening is determined.

Coercivity values are lower than those reported for cobalt particles made using other techniques, such as vapor deposition;[6] however, direct comparisons are difficult because of the tendency for particles exposed to the air to form oxide shells. The core-shell morphology can significantly alter the magnetic behavior.

The coercivities of runs 81 and 82 range from 100 Oe to a maximum of 200 Oe, suggesting that the magnetic behavior of high-temperature-synthesized materials is dependent on factors other than the cobalt DCS. Extensive analysis using models allowing separation of peak-broadening effects due to strain from those due to particle size should help us determine the extent to which strain is responsible for the observed behavior. Other factors that must be considered include the effects of the solvents on the particle structure and composition during synthesis, and the possibility of unexpected compounds interfering with obtaining the DCS. Electron microscopy will be used to determine whether stacking faults are present.

\section{CONCLUSIONS}

High-temperature chemical synthesis produces materials with higher coercivities and remanence ratios than the same synthesis performed at room temperature. Significant differences in magnetic behavior within the group of imaterials synthesized at high-temperatures cannot be attributed solely to dependence on the DCS, as is the case in room-temperaturesynthesized materials. Fcc stacking faults are suggested by differential broadening of the XRD peaks and may be due to the non-equilibrium nature of the synthesis technique. Further structural studies are necessary to elucidate the mechanisms responsible for the unique behavior of these materials.

\section{REFERENCES}

[1] D. L. Leslie-Pelecky and R. D. Rieke, "Magnetic Properties of Nanostructured Materials," Chem. Mater. 8, 1770-1783, 1996.

[2] H. Gleiter, "Nanostructured Materials - Scientific Background and Technological Perspectives," Mat. Sci. Forum 189-190, 67, 1995.

[3] R. D. Rieke, "Preparation of Highly Reactive Metal Powders and their Use in Organic and OrganoMetallic Compounds," Acc, Chem. Res. 10, 301, 1977.

[4] R. D. Rieke, "Preparation of Highly Reactive Powders/Surfaces and their use in the Preparation of Organometallic Compounds," Critical Reviews in Surface Chemistry 1, 131, 1991.

[5] D. L. Leslie-Pelecky, S.-H. Kim, M. Bonder, X, Q. Zhang and R. D. Rieke, "The Structural Properties of Chemically Synthesized Nanostructured $\mathrm{Ni}$ and $\mathrm{Ni}_{3} \mathrm{C}$," accepted to Chem. Mater.

[6] S. Gangopadhyay, G. C. Hadjipanayis, C. M. Sorensen and K. J. Klabunde, "Effect of Particle Size and Surface Chemistry on the Interactions Among Fine Metallic Particles," IEEE Trans. Magn. 29, 2619, 1993. 